\title{
Mécanique du contact rugueux et perception tactile
}

Dans un contact entre solides

rugueux, I'interface constitue

la partie la plus déformable.

Son comportement mécanique

détermine les contraintes

s'établissant dans les deux

solides, ainsi que la dynamique de frottement.

\section{Deux nouvelles méthodes}

expérimentales, fondées

respectivement sur une

observation optique directe

et sur l'utilisation d'un

microcapteur de force MEMS,

permettent de sonder

la mécanique locale

de ces interfaces.

Le dispositif MEMS,

qui est un analogue très

rudimentaire de l'extrémité

du doigt humain,

nous a permis de proposer

un rôle possible des empreintes digitales dans la transduction de l'information tactile.

Julien Scheibert ${ }^{1}{ }^{2}$, Georges Debrégeas ${ }^{1}$, Alexis Prevost ${ }^{1}$ (alexis.prevost@lps.ens.fr) 'Laboratoire de Physique Statistique de l'ENS, CNRS/ENS/Université Paris 6/Université Paris 7 , 24 rue Lhomond, 75231 Paris, France

2Physics of Geological Processes (PGP), University of Oslo, P0 Box 1048 Blindern, 0316 0slo, Norvège

Le frottement solide demeure un phénomène mal compris, en dépit de son importance dans de nombreux domaines $[1,2]$. L'une des difficultés tient à la multiplicité des échelles spatiales mises en jeu. Les contraintes macroscopiques sont contrôlées par la géométrie des objets en contact, tandis que les processus dissipatifs ont lieu dans les premières couches moléculaires en contact. Les échelles intermédiaires ne peuvent cependant être ignorées : en effet, la plupart des surfaces usuelles, même les plus finement usinées, présentent une rugosité aléatoire à l'échelle du micron. L'interface entre deux solides au contact n'est donc généralement pas homogène, mais formée d'un grand nombre de micro-contacts isolés qui supportent l'ensemble de la charge appliquée

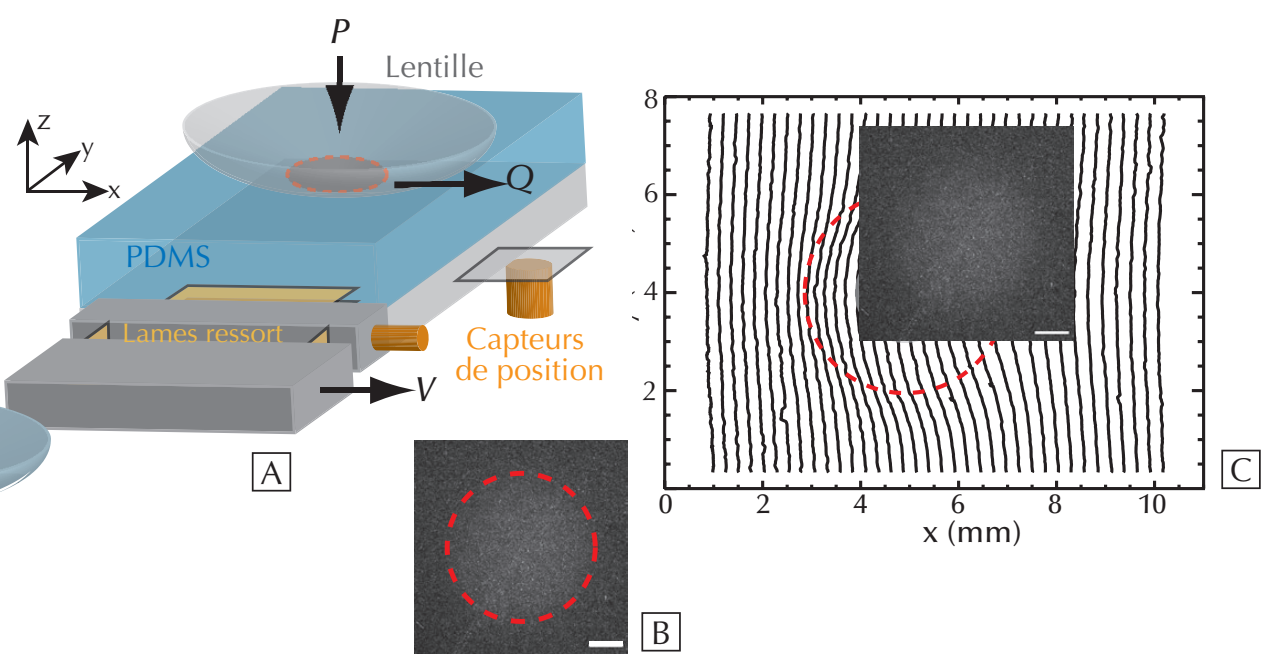

1. Étude optique de l'interface entre un bloc élastomère rugueux et une lentille sphérique en verre.

(A) Schéma du montage expérimental. Le bloc élastomère de polydiméthylsiloxane (PDMS) est solidaire d'un support transparent à travers lequel on éclaire le contact. Ce support est relié à une platine de translation par deux bilames permettant de mesurer, à l'aide de capteurs de position, les charges normale $P$ et tangentielle $Q$ appliquées. La platine, motorisée, permet de déplacer à vitesse constante $V$ le bloc élastomère, tandis que la sphère reste fixe. (B) Image type de l'interface pour $P=0,33 \mathrm{~N}$. Le cercle en pointillés rouges délimite la frontière du contact apparent ; les points blancs dans cette zone sont les microcontacts. La barre blanche mesure $1 \mathrm{~mm}$. (C) Champ des déplacements dans le plan de l'interface rugueuse, mesuré par corrélation d'images au cours d'une charge quasi statique ( $V=4 \mu \mathrm{m} / \mathrm{s}, Q \sim 0,204 \mathrm{~N}$ à l'instant de la mesure). Les déplacements sont représentés par la déformation de lignes initialement rectilignes verticales : ils sont amplifiés d'un facteur 30 pour être visibles (le déplacement maximal sur cette figure est de quelques dizaines de microns). Ces mesures mettent en évidence au sein du contact (frontière en pointillés rouges) la coexistence d'une région centrale collée (déplacement nul), représentée par la zone grisée, et d'une région périphérique de microglissement. 

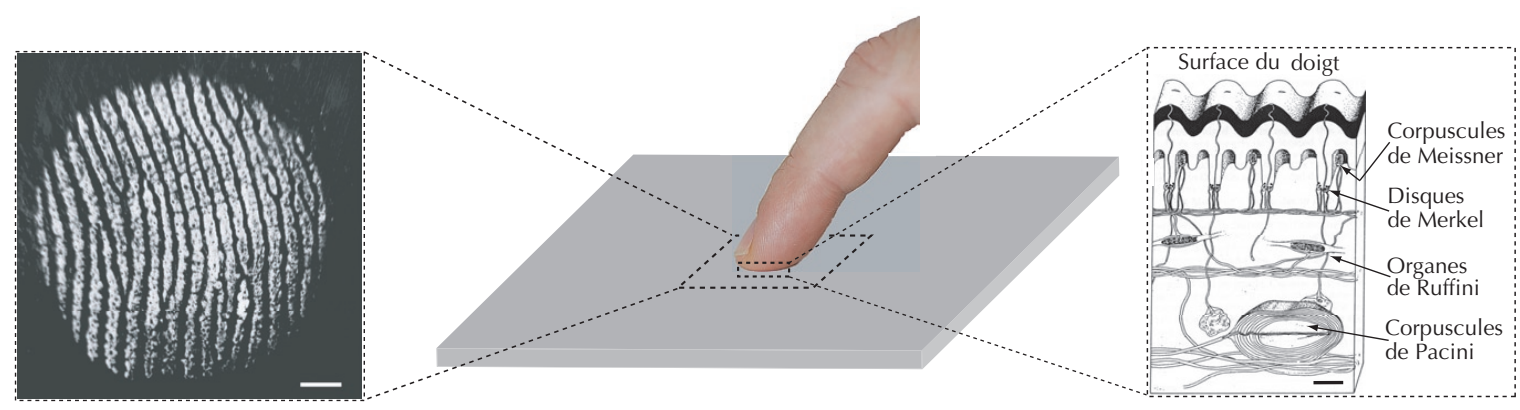

Terminaisons nerveuses

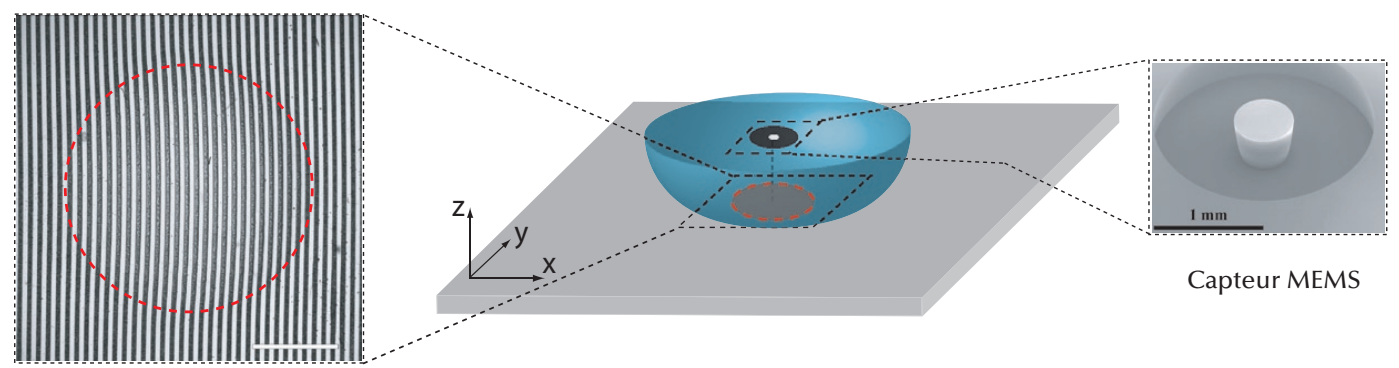

2. Principe de l'approche biomimétique. Panel du haut : l'extrémité du doigt humain est couverte d'empreintes digitales (photo de gauche) et contient un grand nombre de terminaisons nerveuses mécano-réceptrices distribuées sous la surface de la peau jusqu’à des profondeurs de l'ordre de 2 mm (schéma de droite, d’après I. Darian-Smith, Handbook of Physiology, 1984). Panel du bas : pour modéliser le fonctionnement d'un type de ces terminaisons nerveuses (corpuscules de Pacini), un microcapteur de force (CEA-LETI, Grenoble, partie sensible visible sur la photo de droite), a été recouvert d'une calotte sphérique élastique de PDMS d'épaisseur maximale 2 mm. La surface de celle-ci est soit lisse, soit couverte de sillons parallèles analogues aux empreintes digitales (photo de gauche). Pour les deux photos, la barre blanche mesure 2 mm. Ce senseur biomimétique est mis en contact puis frotté contre des lames de verre présentant une rugosité contrôlée de période de l'ordre de quelques dizaines de microns.

$\gg$

\section{Sonder la mécanique des interfaces}

Nous avons étudié les propriétés de ces couches, en développant des mesures in situ fondées sur une observation optique de l'interface multicontacts en lumière transmise (fig. 1A). La figure 1B montre une image typique obtenue entre une lentille de verre sphérique (lisse à l'échelle de quelques nm) et un bloc élastomère transparent dont la surface au contact est rugueuse. Les aspérités micrométriques transmettent la lumière incidente lorsqu'elles sont en contact ; elles la diffusent lorsqu'elles sont hors contact. Nous avons montré que l'intensité moyenne transmise à travers une région donnée de cette interface peut être reliée de façon simple à la pression qui y est appliquée. En analysant les profils d'intensité transmise au sein d'un contact, il devient ainsi possible d'extraire directement les profils de pression et de caractériser leur dépendance avec les propriétés statistiques de la couche rugueuse [3].

Lorsque l'on déplace la sphère lisse à la surface du bloc rugueux, les microaspérités en contact sont d'abord entraînées par la sphère, puis glissent à sa surface sans subir d'altération détectable. Nous avons utilisé les modulations d'intensité lumineuse induites par ces microaspérités comme marqueurs de position pour mesurer, par corrélation d'images, le champ de déplacement dans le plan de l'interface. Nous avons ainsi étudié la transition vers le glissement : dans la géométrie utilisée, celle-ci se produit par croissance d'une zone périphérique glissante qui coexiste avec une zone centrale circulaire collée (fig. 1C), dont le rayon décroît et s'annule lorsque est atteinte la force seuil nécessaire au glissement macroscopique. Ces mesures de haute résolution spatiale, associées à l'utilisation d'un matériau relativement mou, ont également permis de caractériser la déformation élastique de la couche rugueuse avant la mise en glissement associée au fléchissement des aspérités individuelles. Nous avons ainsi pu évaluer un module de cisaillement effectif de cette couche, qui est environ 100 fois plus faible que celui du matériau massif [3].

Dans un contact rugueux macroscopique, l'interface apparait donc comme l'élément le plus déformable, à la fois en compression et en cisaillement. Par rapport à une interface lisse, la rugosité modifie significativement les conditions aux limites mécaniques qui contrôlent la distribution des contraintes dans le volume des solides en regard. Pour mesurer directement ces contraintes, nous avons noyé un microcapteur de force de type MEMS (Micro-Electro-Mechanical Systems) dans le bloc élastique rugueux.
Nous avons utilisé ce dispositif pour mettre en évidence des écarts aux champs prévus dans le cadre d'une loi de frottement d'Amontons-Coulomb ${ }^{(1)}$, attribuables à l'épaisseur finie de cette interface rugueuse [4].

\section{Le toucher : une question de frottement}

La main humaine est un outil exceptionnel, sans équivalent dans le domaine de la robotique. Elle permet de saisir et de manipuler des objets avec une grande précision, mais aussi d'en évaluer certaines propriétés mécaniques ou physiques, telles que la rugosité, la dureté ou le poids. Ces deux fonctions sont rendues possibles par la grande sensibilité tactile de l'extrémité des doigts. Le fonctionnement du système tactile cutané est, à première vue, très rudimentaire : lors de l'exploration tactile d'un objet texturé, les déformations du derme sont traduites en influx nerveux par une population de terminaisons nerveuses mécano-sensibles situées sous l'épiderme, à une profondeur millimétrique (fig. 2) [5] . Pourtant, les performances de ce système perceptif restent pour une large part incomprises et constituent, du point de vue métrologique, un véritable exploit. Ainsi, il permet de détecter et de discriminer 
aisément des textures fines (par exemple différents tissus ou papiers), impliquant des tailles caractéristiques latérales inférieures à $200 \mu \mathrm{m}$, en utilisant des capteurs dont la résolution spatiale est centimétrique !

Dans ce contexte, le dispositif MEMS reproduit de façon très rudimentaire le fonctionnement de l'extrémité du doigt humain : le MEMS et le film d'élastomère qui le recouvre jouent respectivement le rôle d'une terminaison nerveuse et de la peau. Cette approche biomimétique (fig. 2) nous a permis d'étudier les processus mécaniques à l'œuvre dans le toucher humain.

Cet outil nous a permis de comprendre comment les propriétés physiques de la peau et les conditions d'exploration (vitesse de frottement, charge appliquée) contrôlent les caractéristiques de filtrage de l'information tactile. Nous avons en particulier identifié un rôle possible des empreintes digitales dans ce processus, en comparant les signaux de contraintes obtenus en frottant une peau lisse et une peau avec "empreintes " (i.e. dont la surface présente des sillons parallèles réguliers) contre des surfaces planes présentant une rugosité aléatoire contrôlée. Nous avons observé que la présence d'empreintes conduit à une amplification sélective des modulations des contraintes sous-cutanées à la fréquence $V / L$, où $V$ est la vitesse du doigt sur la surface et $L$ la période des empreintes [6]. Dans les conditions typiques d'exploration tactile, cette fréquence est de l'ordre de 200-300 $\mathrm{Hz}$ et correspond au maximum de sensibilité des corpuscules de Pacini (fig. 2, schéma de droite), les mécanorécepteurs impliqués dans la perception des textures fines [7].

Au-delà des enjeux fondamentaux pour la biologie de la perception, cette découverte ouvre des voies nouvelles pour l'amélioration de la sensibilité des capteurs tactiles en robotique.
Références

1 - B.N.J. Persson, Sliding Friction, Springer (2000).

2 • T. Baumberger et C. Caroli, "Solid friction from stick-slip down to pinning and aging", Advances in Physics, 55 (2006) 279-348.

3 -J. Scheibert, Mécanique du contact aux échelles mésoscopiques, Edilivre (2008) (tel-00172935).

$4 \bullet J$. Scheibert, A. Prevost, J. Frelat, P. Rey et $\mathrm{G}$. Debrégeas, "Experimental Evidence of Non-Amontons Behaviour at a Multi-contact Interface", Europhys. Lett., 83 (2008) 34003.

5 - L.A. Jones et S.J. Lederman, Human Hand Function, Oxford University Press (2006).

$6 \bullet J$. Scheibert, S. Leurent, A. Prevost et G. Debrégeas, "The Role of Fingerprints in the Coding of Tactile Information Probed with a Biomimetic Sensor", Science, 323 (2009) 1503.

7 • M. Hollins et S.J. Bensmaïa, "The coding of roughness”, Can. J. Exp. Psych., 61 (2007) 184-195. 